\title{
The Level Set Topological Optimization Method based on the Constraints of Volume and Stress
}

\author{
Dongyue Qu , Fanbing Liu, Xiuming Li and Chunhua Zhao \\ School of Harbin Engineering University, Harbin 150000, China
}

\begin{abstract}
This paper research the topological optimization based on level set method which based on the reaction diffusion equation. The paper propose a Level set topological optimization method which put the compliance as the objective function and take the volume as the main constraint, the element stress as the auxiliary constraint to regulate the volume constraint. This method not only can significantly speed up the optimization process, but can improve the precision of calculation and ensure the stability of the results when meet the stress constraint. Eventually, the proposed method can convenient the level set topological optimization to apply in engineering practice.
\end{abstract}

\section{Introduction}

At present, continuous structure topological optimization has become an important research field of engineering application. The principle of level set method is simple and clear, which won't appear the phenomenon of checkerboard, mesh dependence. So that, this method has a good development prospect.

Level set method was proposed by Osher and Sethian [1] which used to study the dynamic interface tracking as a numerical method. The reaction diffusion equation was introduced to solve the level set function method for structural topological optimization by Masaki Otomori [2]. What's more, the design of structure topological optimization which based on Flexible objective function by Gradient method was proposed by C. G. Zhang and Z. H. Xiong [3]. Moreover, X.Gu introduces some methods of stress to solve the Structural numerical optimization problem [4]. J.H. Rong and S. Ge, G etc proposed a topological optimization method of continuous structure, which combine the displacement method with the analysis of stress sensitivity [5]. ICM method is proposed to solve the problem of topological optimization that Plate and shell with stress constrains by X. D. Liu, Y. K. Sui etc [6].

\section{Math model of topology optimization}

The idea of level set topological optimization is that we establish higher dimension curve or surface of level set function, and then the forms of structural topology was described in the Fig. 1. During the level set topological optimization, we define $\phi$ as the level set function, D as design domain. The positive value of $\phi$ represents the material area and the negative value represents the empty domain. Besides, zero represents the boundary of structure, as shown in Eq. 1

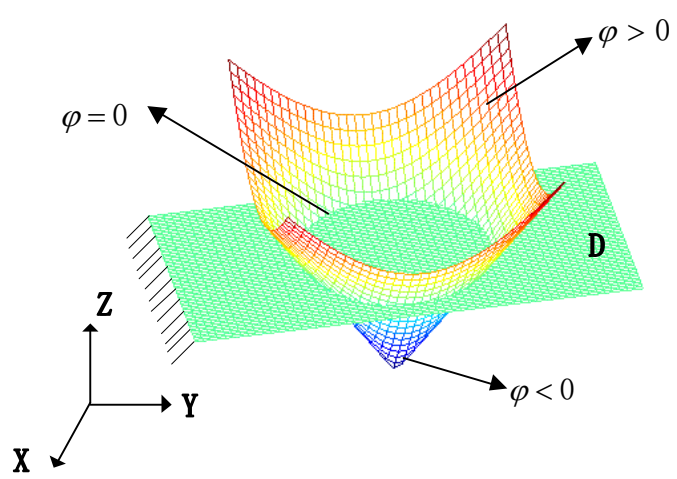

Figure 1. The level set function diagram

$$
\left\{\begin{array}{lr}
\varphi(x)>0 & \text { if } x \in \Omega \\
\varphi(x)=0 & \text { if } x \in \partial \Omega \\
\varphi(x)<0 & \text { if } x \in D / \Omega
\end{array}\right.
$$

\subsection{The derivation formula of topological optimization}

The objective function $J$ of the design is to minimize structural compliance, and get a reasonable structure.

$$
\begin{array}{ll}
\min _{\varphi} & J=\int_{\Gamma} F_{i} u_{i} d \Gamma \\
\text { s.t. } & G=\int_{\Omega} d \Omega-V^{*} \leq 0
\end{array}
$$




$$
\begin{aligned}
& \operatorname{div}\left(E_{i j k l} u_{k . l}\right)=\operatorname{div}\left(\sigma_{i j}\right)=0 \quad \text { on } \Omega . \\
& u_{\mathrm{i}}=0 \quad \text { in } \Gamma_{u} \\
& F_{i}=\bar{F}_{i} \quad \text { in } \Gamma_{f} \\
& \sigma_{m, p} \leq\left[\sigma_{s}\right]
\end{aligned}
$$

where $V^{*}$ is the upper limit of volume constraint which is a shifty parameter, $E_{i j k l}$ is the elastic tensor, $u_{i}$ is displacement of unit, $F_{i}$ is the external force acting at the boundary $\Gamma_{f}$, besides $\Gamma_{u}$ is the boundary of fixed constraint and its value equal to $0, \operatorname{div}\left(E_{i j k l} u_{k, l}\right)=0$ represent that there is no other external load on the material area. What's more, $p$ are the iterations, $\sigma_{m, p}$ represent maximum unit stress at $p$ times. $\left[\sigma_{s}\right]$ represents the allowable stress. Using Lagrange multiplier algorithm transform above formulas into a unconstrained Lagrange formula is shown as follows:

$$
\bar{J}=\int_{\Gamma} F_{i} u_{i} d \Gamma+\int_{\Omega}^{\sim} u_{i} d i v\left(E_{i j k l} u_{k, l}\right) d \Omega+\lambda\left(\int_{\Omega} d \Omega-V^{*}\right)
$$

where $J$ represents the new objective function, $\mathrm{u}_{i}$ and $\lambda$ represents the Lagrange multipliers [2].

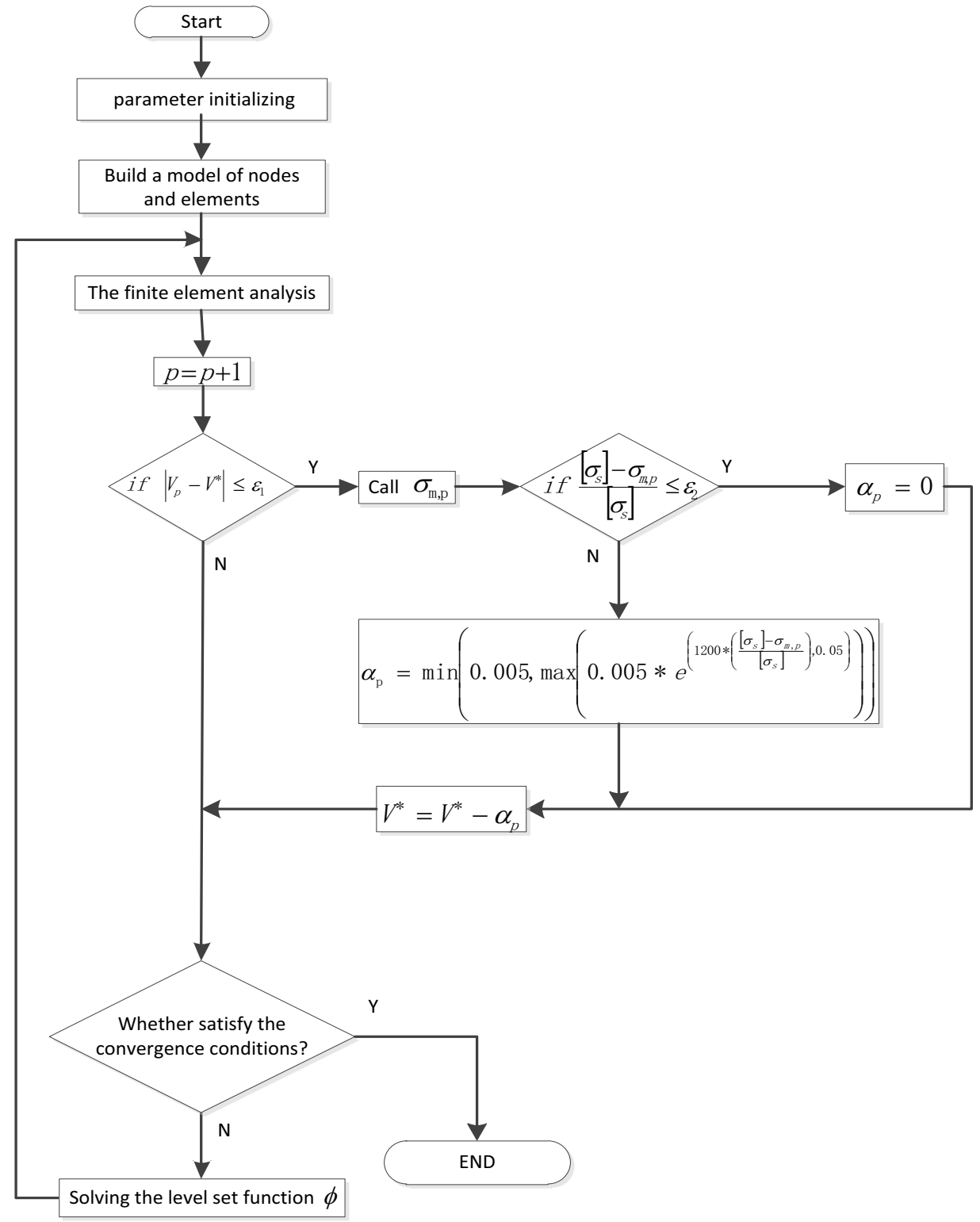

Figure 2. Flow diagram of the bidirectional improvement

\subsection{Volume constraints formula}

Volume constraint is applied to controls the changed volume of material. The details of solve are shown as follows:

$$
V^{*}=V^{*}-\alpha_{p}
$$

where, the solve of $\alpha_{p}$ as follows: 


$$
\begin{gathered}
\text { If }\left[\sigma_{s}\right]-\sigma_{m, p} \leq \varepsilon_{2}\left[\sigma_{\mathrm{s}}\right] \\
\alpha_{p}=0 \\
\text { If }\left[\sigma_{s}\right]-\sigma_{m, p}>\varepsilon_{2}\left[\sigma_{\mathrm{s}}\right] \\
\alpha_{p}=\min \left(0.005, \max \left(0.005 * \mathrm{e}^{\left(1200 *\left(\frac{\left[\sigma_{s}\right]-\sigma_{\mathrm{m}, p}}{\left[\sigma_{\mathrm{s}}\right]}\right), 0.05\right)}\right)\right) .
\end{gathered}
$$

As shown in Eq. 5. We can see that $\alpha_{p}$ represent the value of $\alpha$ in $p$ times. With the change of unit stress, the value of $V^{*}$ is also changed which is the main constrain that can control the decrease of the material. Besides, the value of $V^{*}$ is decided by $\alpha_{p}$. What's more, the drop speed of volume constrain $V^{*}$ will increase when the maximum stress value fully meet the located conditions. On the other hand, the drop speed of volume constrain $V^{*}$ will decrease when the maximum stress value will meet the located conditions. From that, we can improve the accuracy of calculation.

\subsection{The computational formula of stress}

In this paper, the stress calculation formula as follows:

$$
\sigma_{\mathrm{i}}=\mathrm{DBu}_{\mathrm{i}}
$$

where D represent the elastic matrix, $\mathbf{B}$ represent the strain matrix, besides, the $\mathbf{u}_{\mathbf{i}}$ represent the vector of node displacement in element $i$.

The stress vector value of element $i$ can be represented as $\boldsymbol{\sigma}_{\mathbf{i}}=\left(\boldsymbol{\sigma}_{\mathbf{x}}, \boldsymbol{\sigma}_{\mathbf{y}}, \boldsymbol{\tau}_{\mathbf{x y}}\right)^{\mathbf{T}}$, and its Michelle unit stress is shown in Eq. 7.

$$
\begin{gathered}
\sigma_{\mathrm{i}}=\sqrt{\sigma_{x}^{2}+\sigma_{y}^{2}-\sigma_{x} \sigma_{y}+3 \tau_{x y}^{2}}=\left(\boldsymbol{\sigma}_{\mathbf{i}}^{\mathrm{T}} \mathbf{T} \boldsymbol{\sigma}_{\mathbf{i}}\right)^{\mathbf{1 / 2}} \\
\mathbf{T}=\left[\begin{array}{ccc}
1 & -0.5 & 0 \\
-0.5 & 1 & 0 \\
0 & 0 & 3
\end{array}\right]
\end{gathered}
$$

where $\sigma_{X}, \sigma_{y}$ respectively represent the normal stress along with the direction of $X$ and $Y ; \boldsymbol{\tau}_{\mathbf{x y}}$ represent the shear stress. Besides, $\mathbf{T}$ is regarded as the Coefficient matrix.

The flow diagram of stress constrain is shown as Fig. 2. In the Fig. 2, $\varepsilon_{1}$ and $\varepsilon_{2}$ are the error parameters, $\varepsilon_{1}$ is equal to $0.01, \varepsilon_{2}$ is equal to 0.00004 .

\section{Numerical examples}

Now, Here will prove the effectiveness and accuracy of level set topological optimization which based on the stress and volume constrains. The constrain and force is applied in a cantilever beam as shown in Fig. 3, besides the thickness value of the plate is one millimeter, and Young's modulus value of the material is $E=2.1 * 10^{11} \mathrm{pa}$, what's more, The value of poisson's ratio is equal to 0.3 .

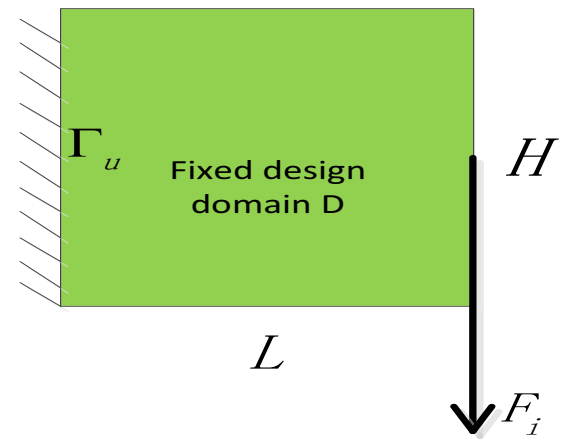

Figure 3. Structure diagram of the cantilever beam

\subsection{Example 1}

The wide $\mathrm{L}$ of the structure is equal to $80 \mathrm{~mm}$, high $\mathrm{H}$ is $64 \mathrm{~mm}$, and concentrated load $F_{\mathrm{i}}$ is equal to $3500 \mathrm{~N}$. The allowable value of stress is equal to $218 \mathrm{MPa}$; the result of example 1 is shown in Fig. 4 and Fig. 5.

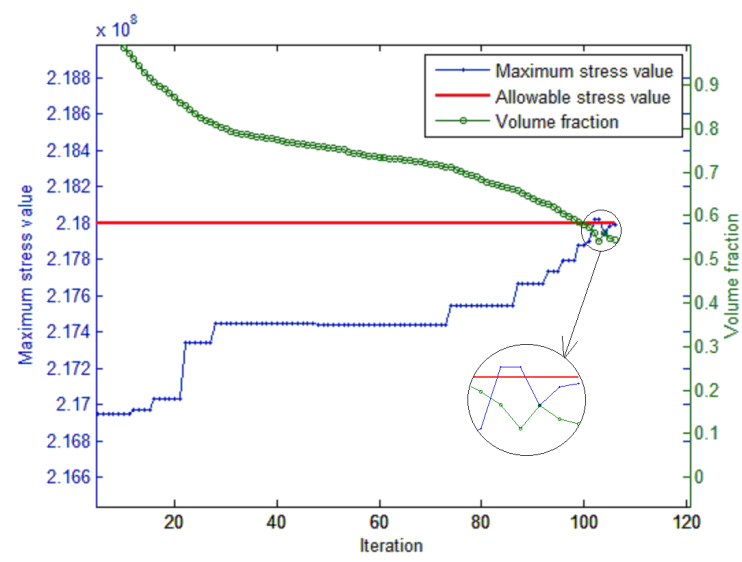

Figure 4. Variation related of parameters in example 1

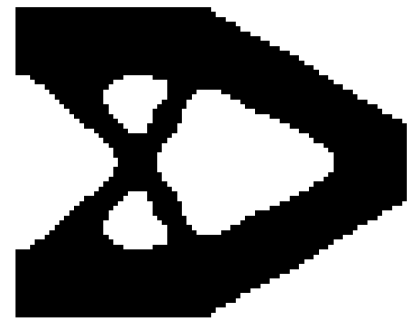

Figure 5. Topological structure diagram of example 1

As shown in Fig. 4, we can see that blue line represents the maximum stress value in each iteration of the structure, red one represents the allowable stress 
value and the green line represents the rest of the volume fraction. Along with the iteration, the volume fraction decreased continuously, the maximum stress value of structure increased continuously and it will approach the allowable stress values.

After calculating, we can know that the volume fraction of the structure is 0.536 , and the stress value is shown as $\sigma_{m}=217.98 \mathrm{MPa}<\left[\sigma_{s}\right]$, which meet the condition that stress is not more than allowable stress.

\subsection{Example 2}

The wide $\mathrm{L}$ of the structure is equal to $40 \mathrm{~mm}$, high $\mathrm{H}$ is $80 \mathrm{~mm}$, and concentrated load $F_{\mathrm{i}}$ is equal to $4000 \mathrm{~N}$. The allowable value of stress is equal to $249 \mathrm{MPa}$; the result of example 2 is shown in Fig. 6 and Fig. 7.

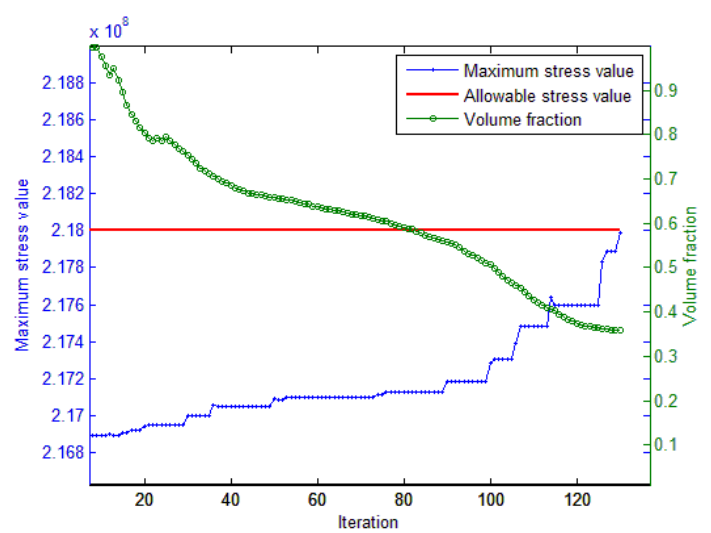

Figure 6. Variation related of parameters in example 2

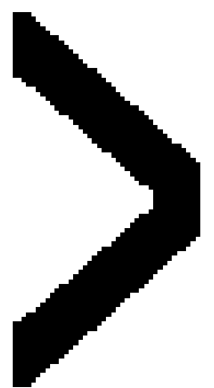

Figure 7. Topological structure diagram of example 2

As shown in Fig. 6, we can see that blue line represents the maximum stress value in each iteration of the structure, red one represents the allowable stress value and the green line represents the rest of the volume fraction. Along with the iteration, the volume fraction decreased continuously, the maximum stress value of structure increased continuously and it will approach the allowable stress values.
After calculating, we can know that the volume fraction of the structure is 0.358 , and the stress value is equal to $\sigma_{m}=248.995 \mathrm{MPa}<\left[\sigma_{s}\right]$, which meet the condition that stress is not more than allowable stress.

\section{Summary}

This paper introduce the stress constraint to the level set method for topological optimization, and set a changed parameters as the threshold value to regulate the volume of structure, which based on the relationship between the located allowable stress and the of unit stress at every iteration. What's more, the parameters not only speed up the optimization process when the maximum stress value fully meet the located conditions, but also can slow down the optimize process, improve the accuracy of calculation, ensure stability of the results when the maximum stress value will meet the located conditions. Eventually, the proposed method can convenient the level set topological optimization to apply in engineering practice.

\section{References}

1. S. Osher and J.A. Sethian: Fronts propagating with curvature dependent speed: algorithms based on Hamilton-Jacobi formulations. J. Comput. Phys. 79 pp. 12-49, (1988).

2. M.Otomori, T. Yamada, K. Izui and S. Nishiwaki: Matlab code for a level set-based topology optimization method using a reaction diffusion equation. Struct. Multidiscip.O 51, pp. 1159-1172, (2015).

3. C. G. Zhuang, Z. H. Xiongand D. Han:Structural Topology Optimization Based on Level Set Method and von Mises Stress, (2006).

4. W.S. Zhang, X. Guo, M.Y. Wang and P. Wei: Optimal topology design of continuum structures with stress concentration alleviation via level set method. Int. J. Numer. Methods Eng., 93, pp. 942959, (2013).

5. J. H. Rong, S. Ge, G. Deng, X. J. Xin and Z. J. Zhao: Structural topological optimization based on displacement and stress sensitivity analyses. Chinese Journal of Theoretical and Applied Mechanics. 4, pp. 0518-0529, (2009).

6. X.D. Liu, Y.K. Sui and H.P. Yu: Topological Optimization of plate and shell structure with stress constraints. Mechanics in Engineering. 4, pp. 035039, (2012). 